\title{
Psychosocial factors associated with risk of suicidal recurrence in suicidal patients
}

\author{
1,2L Ghanmi, ${ }^{1} \mathrm{M}$ Elbehi, ${ }^{1} \mathrm{~W}$ Abbes, ${ }^{1} \mathrm{~B}$ Chayeb, ${ }^{1} \mathrm{D}$ Farhat, ${ }^{1} \mathrm{M}$ Abbes, ${ }^{2,3} \mathrm{M}$ Maalej, ${ }^{2,3} \mathrm{Nada}$ \\ Charfi, ${ }^{1} \mathrm{R}$ Ben Fradj, ${ }^{2,3} \mathrm{~L}$ Zouari, ${ }^{2,3} \mathrm{M}$ Maalej
}

${ }^{1}$ Psychiatry Department, Regional Hospital of Gabes. Tunisia

${ }^{2}$ Faculty of Medicine of Sfax. Tunisia

${ }^{3}$ Department of psychiatry «C», UMC Hedi Chaker of Sfax. Tunisia

\section{Introduction:}

* Although several factors are associated with suicidal behavior, it appears that some psychosocial factors predispose subjects to suicide attempts (SA).

\section{Aims:}

* To determine the psychosocial factors associated with suicidal recurrence in suicidal patients followed at the outpatients' psychiatry department in Gabes (southern Tunisia).

\section{Patients and Methods:}

* This was a retrospective, descriptive and analytical study.

* The study included all the patients who had attempted suicide and who were referred to the psychiatric department of the Gabes regional hospital (from 1/1/2009 to 31/12/2015).

* We used a pre-established sheet that explored socio-demographic and family characteristics of suicidal patients.

\section{Results:}

* We have collected 124 patients. (Table 1: socio-demographic and psychosocial characteristics of suicidal patients)

- Suicidal recurrence accounted for $17.8 \%$ of the patients. It was most often early, occurring within six months of a SA in half of the cases.

* The multivariate analysis showed that only the presence of a psychiatric history in one of the parents was a determinant of the risk of suicidal recurrence $(\mathrm{OR}=6.9$ [1.2-38], $\mathrm{P}=0.02)$. (Table 2: Risk factors for recurrence)

\section{Discussion:}

* The existence of risk factors does not necessarily lead to a suicidal behaviour [1]. Generally, a combination of several stress factors increases the individual's vulnerability to suicidal behaviour. It is therefore important to identify high-risk people in any community in order to help develop strategies to prevent suicidal behaviour.

* Suicidal recidivism is estimated at about $25 \%$ of cases [2], with a higher risk during the first year following the SA: the first 6 months appear to be the period of most recurrence risk [3] with even a higher risk during the first 2 months [4]

\begin{tabular}{|l|c|c|}
\hline \multicolumn{1}{|c|}{ Characteristic } & $\%$ & Mean \\
\hline Age (years) & & 25 \\
\hline Female & 82.3 & \\
\hline Single & 82.3 & \\
\hline Mental disorder in the parents & 19.7 & \\
\hline One or more psychosocial risk factors : & 66 & \\
Academic and professional difficulties: & 26.2 & \\
Financial difficulties : & 24.6 & \\
Unemployment : & 14.8 & \\
\hline Suicidal recurrence : & $17.8 \%$ & \\
\hline
\end{tabular}

Table 1: Socio-demographic and psychosocial characteristics of suicidal patients

\section{Risk factors for recurrence:}

* Depressive disorders [5,6,7,8] with the notion of despair [5] The existence of even isolated depression is also described in many studies as a risk factor for recurrence [3]

Feelings of hopelessness have even been considered as a risk factor for suicidal recurrence $[9,10]$.

As well as the persistence of suicidal thoughts after the first attempt

[11] and the persistence of a thymic disorder at the end of hospitalization

* Personal history of SA [6].

* Family pathological history [9], particularly depression, SA [5] and alcoholism [12] increase the risk of suicidal recurrence.

* Other co-morbidities [9,10] :The existence of psychosis and taking treatments for a psychic disorder [6,7] are risk factors for suicidal recurrence [3]. Anxiety disorders associated with a history of SA increase the risk of recurrence [3]. Taking treatment for a mental disorder or for the first SA is also a risk factor for recurrence $[9,10]$. Chronic somatic diseases also influence suicidal recurrence [13].

- Personality disorder has a positive predictive value for suicidal recurrence: borderline [5.8] , antisocial [3]

* Alcohol dependence [6], misuse of toxic substances in men [7].

- Poor physical health [14].

* A history of trauma in early childhood [7,8] or sexual abuse[15,16] are correlated with repetition of the passages to the suicidal act.

\section{Conclusion:}

Special attention should be paid to the family history of mental disorders and unemployment when assessing the risk of suicidal recurrence in our patients.

\begin{tabular}{|c|c|c|c|c|}
\hline & \multicolumn{2}{|c|}{ Recurrence } & \multirow[t]{2}{*}{$\mathbf{P}$} & \multirow[t]{2}{*}{ OR } \\
\hline & Yes (\%) & No (\%) & & \\
\hline Male & 36.4 & 13.7 & 0.026 & 3.5 \\
\hline Unemployment & 31 & 13.7 & 0.035 & 2.8 \\
\hline $\begin{array}{l}\text { Presence of a mental disorder in one } \\
\text { of the parents }\end{array}$ & 33.3 & 14.6 & 0.05 & 2.9 \\
\hline Divorce of parents & 66.7 & 13.9 & 0.001 & 12.3 \\
\hline Bipolar disorder & 47.1 & 13.1 & 0.002 & 5.9 \\
\hline Personality disorder & 44.4 & 15.7 & 0.05 & 4.3 \\
\hline
\end{tabular}

Table 2: Factors associated with suicidal recurrence 